\title{
Active Millimeter-Wave Radiometry for Nondestructive Testing/Evaluation of Composites--Glass Fiber Reinforced Polymer
}

DOI:

10.1109/TMTT.2016.2625785

\section{Document Version}

Accepted author manuscript

Link to publication record in Manchester Research Explorer

Citation for published version (APA):

Viegas, C., Alderman, B., Huggard, P. G., Powell, J., Parow-Souchon, K., Firdaus, M., Liu, H., Duff, C. I., \& Sloan, R. (2016). Active Millimeter-Wave Radiometry for Nondestructive Testing/Evaluation of Composites--Glass Fiber Reinforced Polymer. IEEE Transactions on Microwave Theory and Techniques. https://doi.org/10.1109/TMTT.2016.2625785

Published in:

IEEE Transactions on Microwave Theory and Techniques

\section{Citing this paper}

Please note that where the full-text provided on Manchester Research Explorer is the Author Accepted Manuscript or Proof version this may differ from the final Published version. If citing, it is advised that you check and use the publisher's definitive version.

\section{General rights}

Copyright and moral rights for the publications made accessible in the Research Explorer are retained by the authors and/or other copyright owners and it is a condition of accessing publications that users recognise and abide by the legal requirements associated with these rights.

\section{Takedown policy}

If you believe that this document breaches copyright please refer to the University of Manchester's Takedown Procedures [http://man.ac.uk/04Y6Bo] or contact uml.scholarlycommunications@manchester.ac.uk providing relevant details, so we can investigate your claim.

\section{OPEN ACCESS}




\title{
Active Millimeter-Wave Radiometry for Non-Destructive Testing/Evaluation of Composites - Glass Fiber Reinforced Polymer
}

\author{
Colin Viegas, Student Member, IEEE, Byron Alderman, Peter G. Huggard, Senior Member, IEEE, Jeff \\ Powell, Kai Parow-Souchon, Muhammad Firdaus, Student Member, IEEE, Hairui Lui, Christopher I. \\ Duff, Member, IEEE, and Robin Sloan, Senior Member, IEEE
}

\begin{abstract}
Manufacture and in-service assessment of composite materials can be challenging since there is, yet, no standard method for testing them. Nevertheless, regular inspection is necessary to maintain structural integrity. This paper describes a non-destructive, broadband noise mapping method that uses a millimeter-wave radiometer to detect defects in composite materials. The $W$-band system is configured for transmission mode measurement in which an amplified photonic source illuminates a glass fiber reinforced polymer (GFRP) containing machined defects. A high sensitivity radiometer is used for imaging the sample. The radiometer consists of a Schottky diode -based frequency tripler and fundamental mixer.
\end{abstract}

Index Terms-Composite material, fiber reinforced polymer, millimeter wave, mixer, non-destructive, photonic, radiometer, Schottky diode, sensitivity.

\section{INTRODUCTION}

G LASS fiber is one of the most commonly used materials for reinforcing polymers. Other materials include carbon fiber and kevlar which regularly find wide applications in the automotive, aerospace and construction sectors. Glass fiber reinforced polymers (GFRP) are known to be strong, lightweight, and ductile and hence are successfully used for reinforcements [1]. Composite materials can develop cracks, delaminations, and voids and demand maintenance determined by imaging techniques to avoid structural deterioration and possible failure.

Non-destructive testing/evaluation (NDT/E) is routinely used for the inspection of flaws in composite materials. Several established methods have been used with varying success [2]. Methods such as radiography, ultrasonic, acoustic, $\mathrm{X}$-ray, vibration, eddy current, etc., are commonly used in industrial NDT/E. Ultrasonic methods can suffer from a poor

Manuscript received January 15, 2016; revised May 04, 2016 and August 25, 2016; accepted October 01, 2016.

C. Viegas, M. Firdaus A. J. K, R. Sloan, C. I. Duff are with The University of Manchester, Manchester, UK, M13 9PY.

(email: colin.viegas@postgrad.manchester.ac.uk).

B. Alderman, J. Powell and H. Lui are with Teratech Components Ltd., Rutherford Appleton Lab., Harwell Oxford, UK, OX11 0QX.

P. G. Huggard and K. Parow-Souchon are with Millimetre Wave Technol. Group, Rutherford Appleton Lab., Harwell Oxford, Didcot, UK, OX11 0QX. signal-to-noise ratio due to high attenuation, while X-rays do not always provide a good contrast between defect and nondefect areas [3]. The use of electromagnetic (EM) radiation for investigation offers considerable benefits when evaluating a structure for deformities. This method does not require probe contact with the surface or couplant and can yield a depth of penetration dependent on the material loss tangent and operating frequency. The measurement resolution improves with increasing frequency enabling the inspection of defects in the sub-mm range at $\mathrm{THz}$.

Within the millimeter-wave (mm-wave) band of inspection, the use of a waveguide probes is quite popular [4]-[6]. In this method, the near field sample interaction is measured with a waveguide and a network analyzer as the wave propagating in the material is reflected and scattered by discontinuities in the dielectric characteristics. Techniques such as dielectrically loading an open-ended waveguide can be used to reduce the waveguide size while maintaining resolution [3]. Active thermographic NDT reported in [5], [7], illuminates the sample and detects the differential temperature change using a thermal camera. Other EM methods include free space microwave NDT using horn lens antennas with a network analyzer [8] and the use of inter-digit sensor to measure the Sparameters along a defected surface [9]. Polarization studies on a GFRP sample using a synthetic aperture radar technique is reported in [10].

Table I and II presents an overview of some specific research published in the field of NDT/E. Table I compares research based on the technique used for non-destructive testing of a composite sample. For simplicity, only techniques used to image GFRP samples have been included. Also, methods like the ones included in the table have been omitted. Table II compares research based on applications where a $W$ band system has been used for inspection. The active mmwave inspection method presented in this paper represents a new approach for inspection of composite materials in comparison to the existing research. This technique for inspection combines two different research fields which presents a potentially new and interesting area for study.

In this method, a broadband photonic source generating random mm-wave noise is quasi-optically projected onto the sample. Transmitted signal variations due to sample inhomogeneity are detected by the $W$-band radiometer as it is 
TABLE I

OVERVIEW OF EM IMAGING TECHNIQUES USED FOR NDT/E OF GFRP

\begin{tabular}{|c|c|c|c|c|}
\hline NDT/E technique & Frequency $(\mathrm{GHz})$ & TX/RX Scheme & Field Analysis & Reference \\
\hline $\begin{array}{l}\text { Inverse synthetic aperture } \\
\text { radar (ISAR) }\end{array}$ & $8-18$ & Radar feed-horn & Far field & Buyukozturk et al. [11] \\
\hline $\begin{array}{l}\text { Frequency Modulated } \\
\text { Continuous Wave (FMCW) } \\
\text { radar }\end{array}$ & $75-110$ & Radar feed-horn & Near field & Dobmann et al. [12] \\
\hline $\begin{array}{l}\text { Active microwave } \\
\text { thermography }\end{array}$ & 2.4 & Horn/thermal camera & Near field & Foudazi et al. [7] \\
\hline $\begin{array}{l}\text { Terahertz time-domain } \\
\text { spectroscopy }\end{array}$ & $0-3000$ & $\begin{array}{c}\text { Femtosecond } \\
\text { laser/photoconductive dipole } \\
\text { antennas }\end{array}$ & - & Richter et al. [13] \\
\hline Infrared thermography & - & $\begin{array}{c}\text { Photographic flashes/IR } \\
\text { camera }\end{array}$ & - & $\begin{array}{c}\text { Ibarra-Castanedo et al. } \\
{[14]}\end{array}$ \\
\hline $\begin{array}{l}\text { Synthetic aperture radar } \\
\text { (SAR) }\end{array}$ & $75-110$ & Radar feed-horn & - & Nezadal et al. [10] \\
\hline Microwave heating & $\begin{array}{c}\text { Low power- } 18, \text { High } \\
\text { power- } 2.54\end{array}$ & Waveguide/thermal camera & Far field & Cheng et al. [5] \\
\hline $\begin{array}{l}\text { Active millimeter-wave } \\
\text { radiometry }\end{array}$ & $75-110$ & Noise source/radiometer & Far field & Present system \\
\hline
\end{tabular}

TABLE II

OVERVIEW OF mm-WAVE SYSTEMS USED FOR VARIOUS NDT/E APPLICATIONS

\begin{tabular}{|c|c|c|c|}
\hline NDT/E Application & System & $\begin{array}{c}\text { Sensitivity }(\mathrm{K}) @ 0 \\
\text { integration time }\end{array}$ & Reference \\
\hline $\begin{array}{l}\text { Detection of space shuttle tile } \\
\text { defects }\end{array}$ & Passive & $0.3 @ 1 \mathrm{~s}$ & Clancy et al. [15] \\
\hline $\begin{array}{l}\text { Inspection of bonding quality in } \\
\text { polymers }\end{array}$ & Active & - & Bakhtiari et al. [16] \\
\hline $\begin{array}{l}\text { Detection of carbon in soot } \\
\text { collection filters }\end{array}$ & Active & - & Shibuya et al. [17] \\
\hline $\begin{array}{l}\text { Corrosion detection in aircraft } \\
\text { structures }\end{array}$ & Active & - & Seah et al. [18] \\
\hline $\begin{array}{l}\text { Monitor healing of burn wounds }{ }^{1} \\
\text { Monitoring of vital signs }{ }^{1}\end{array}$ & $\begin{array}{l}\text { Passive } \\
\text { Active }\end{array}$ & $0.2 @ 1 \mathrm{~s}$ & $\begin{array}{l}\text { Harmer et al. [19] } \\
\text { Bakhtiari et al. [20] }\end{array}$ \\
\hline Inspection of defects in materials & Active & $0.53 @ 3.3 \mathrm{~ms}$ & Present system \\
\hline
\end{tabular}

raster scanned along the focal plane. Signals passing through the GFRP sample undergo scattering from edges, as well as constructive and destructive interference from material weavings or multiple layers. An important advantage of using a broadband imaging system is the inherent averaging of standing waves across the operational bandwidth which results for interfaces [21].

\section{ELECTROMAGNETIC WAVE INTERACTION AT BOUNDARIES OF DIFFERENT MEDIUMS}

A general representation of a plane wave normally incident at an interface is shown in Fig. 1. We assume the wave is propagating in the $+z$ direction. The medium is considered lossless $(\alpha=0, \beta=k)$. If the medium was lossy, the propagation constant would be $\gamma=\alpha+\beta$. The permittivity of medium 1 and medium 2 are $\varepsilon_{1}$ and $\varepsilon_{2}$, respectively, while the permeability, $\mu_{0}$ of both the mediums is 1 . When a wave travelling through medium 1 is incident at the boundary of the medium 2, part of the wave is transmitted while some part is reflected. The electric fields for the incident, reflected, and transmitted, respectively are given below

$$
\begin{aligned}
& E_{y, i}(z, t)=A_{i} e^{j\left(w t-k_{1} z\right)} \\
& E_{y, r}(z, t)=B_{r} e^{j\left(w t+k_{1} z\right)} \\
& E_{y, t}(z, t)=A_{t} e^{j\left(w t-k_{2} z\right)}
\end{aligned}
$$

where $k_{1}$ and $k_{2}$ are the wave numbers for medium 1 and medium 2, respectively. The wave number can be calculated as $k=\omega \sqrt{\varepsilon_{r}}$ where $\varepsilon_{r}$ is the complex relative permittivity. The constant terms $A$ and $B$ represent wave propagation in the $+z$ and $-z$-directions, respectively. The wave impedance of the propagating wave is $Z=\sqrt{\mu / \varepsilon}$. The electric and magnetic fields are related through the wave impedance as $Z=E / H$. Since the tangential component of the electric field 
and magnetic fields remains continuous at the boundary $(z=0)$, we can write

$$
\begin{gathered}
E_{y, i}(z=0, t)+E_{y, r}(z=0, t)=E_{y, t}(z=0, t) \\
H_{x, i}(z=0, t)+H_{x, r}(z=0, t)=H_{x, t}(z=0, t)
\end{gathered}
$$

From the boundary conditions shown above, we obtain

$$
\begin{aligned}
& A_{i}+B_{r}=A_{t} \\
& \frac{A_{i}}{Z_{1}}-\frac{B_{r}}{Z_{1}}=\frac{A_{t}}{Z_{2}}
\end{aligned}
$$

The reflection coefficient, $\Gamma$ and transmission coefficient, $T$ can be obtained from [22], as shown below

$$
\begin{gathered}
\Gamma=\frac{B_{r}}{A_{i}}=\frac{Z_{2}-Z_{1}}{Z_{1}+Z_{2}} \\
\mathrm{~T}=\frac{A_{t}}{A_{i}}=1+\Gamma=\frac{2 Z_{2}}{Z_{1}+Z_{2}}
\end{gathered}
$$

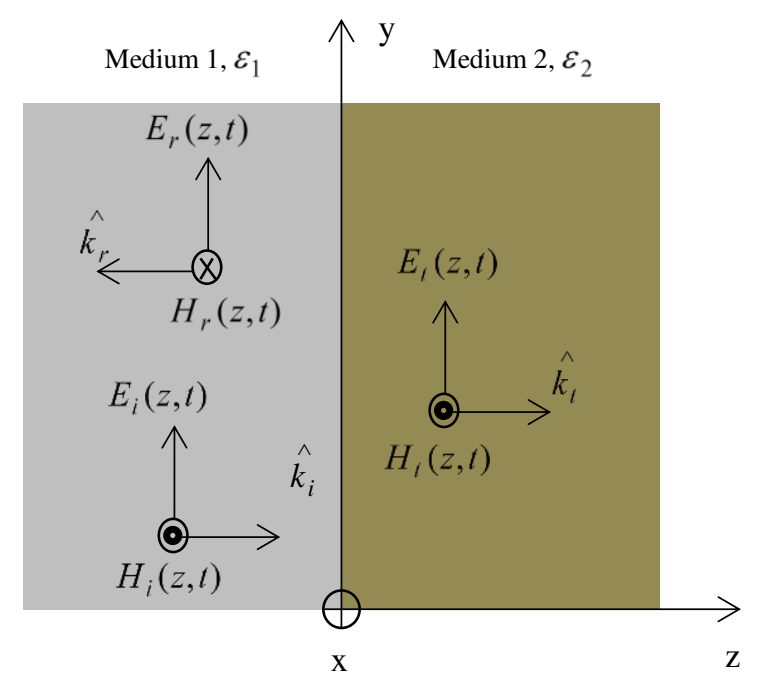

Fig. 1. Transmission and reflection from a general interface at normal incidence.

A normally incident EM wave at an air-dielectric-air interface is illustrated in Fig. 2. The permittivity of air and dielectric mediums are $\varepsilon_{0}$ and $\varepsilon_{r}$, respectively. The permeability, $\mu_{0}$ of both mediums is 1 . At the first interface, the wave is partially transmitted into the next medium and partially reflected. The phase of individual terms when summed will be different due to the phase difference between the dielectric-air boundary. This additional phase difference can be represented as $k_{2} d$ after crossing the interface. We assume the reflection coefficient from the air-dielectric interface is $\Gamma_{1}$ in the $+z$ direction and $-\Gamma_{1}$ in the $-z$ direction. Since medium 3 is identical to medium $1, \Gamma_{2}=-\Gamma_{1}$ and $-\Gamma_{2}=\Gamma_{1}$. Similarly, the transmission coefficient through the air-dielectric interface is assumed as $\mathrm{T}_{1}^{+}=1+\Gamma_{1}$ in the $+z$ direction and $\mathrm{T}_{1}^{-}=1-\Gamma_{1}$ in the $-z$ direction. The total reflected electric field is obtained as shown

$$
E_{r}(z)=E_{i}\left[\Gamma_{1}+\mathrm{T}_{1}^{+} \mathrm{T}_{1}^{-} \Gamma_{2} e^{-j 2 k_{2} d}+\mathrm{T}_{1}^{+}\left(-\Gamma_{2} \Gamma_{1}\right) \mathrm{T}_{1}^{-} \Gamma_{2} e^{-j 4 k_{2} d}+. .\right]
$$

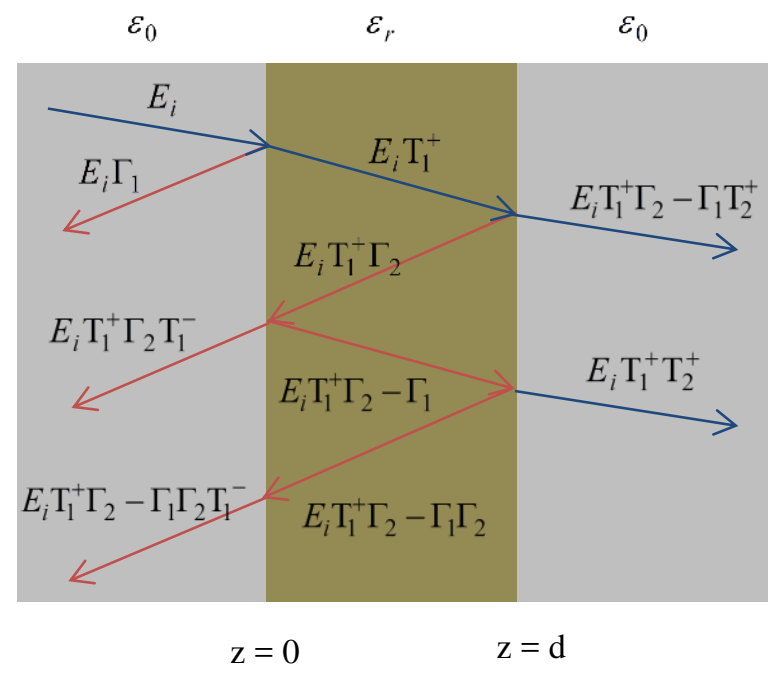

Fig. 2. Transmission and reflection from an air-dielectric-air interface.

$$
E_{r}(z)=E_{i}\left[\Gamma_{1}-\Gamma_{1}\left(1-\Gamma_{1}^{2}\right) e^{-j 2 k_{2} d}\left\{1+\Gamma_{1}^{2} e^{-j 2 k_{2} d}+. .\right\}\right]
$$

The total reflection coefficient is

$$
\begin{gathered}
\Gamma=\frac{E_{r}(z)}{E_{i}}=\Gamma_{1}\left[1-\frac{\left(1-\Gamma_{1}^{2}\right) e^{-j 2 k_{2} d}}{1-\Gamma_{1}^{2} e^{-j 2 k_{2} d}}\right] \\
\Gamma=\frac{E_{r}(z)}{E_{i}}=\frac{\Gamma_{1}\left(1-e^{-j 2 k_{2} d}\right)}{1-\Gamma_{1}^{2} e^{-j 2 k_{2} d}}
\end{gathered}
$$

In a similar way, the total transmitted electric field can be obtained from Fig. 2 as follows

$$
\begin{gathered}
E_{t}(z)=E_{i}\left[\mathrm{~T}_{1}^{+} \mathrm{T}_{1}^{-} e^{-j k_{2} d}+\mathrm{T}_{1}^{+}\left(-\Gamma_{2} \Gamma_{1}\right) \mathrm{T}_{2}^{-} e^{-j 3 k_{2} d}+. .\right] \\
E_{t}(z)=E_{i}\left[\left(1-\Gamma_{1}^{2}\right) e^{-j k_{2} d}\left\{1+\left(\Gamma_{1}^{2} e^{-j 2 k_{2} d}\right)+\left(\Gamma_{1}^{2} e^{-j 2 k_{2} d}\right)^{2}+. .\right\}\right]
\end{gathered}
$$

The total transmission coefficient is

$$
\mathrm{T}=\frac{E_{t}(z)}{E_{i}}=\frac{\left(1-\Gamma_{1}^{2}\right) e^{-j k_{2} d}}{1-\Gamma_{1}^{2} e^{-j 2 k_{2} d}}
$$

Fiber reinforced composites usually exhibit anisotropy and hence the characterization of its material properties depend on the orientation of the measurement plane [22]. For an incoming wave that is obliquely incident, reflections from an anisotropic and heterogeneous material surface are not specular. Scattering of the incident wave from these surfaces depend on properties such as surface roughness, angle of incidence, and material loss [23]. Diffuse reflection from rough surfaces cause the field to scatter in different directions and are rather difficult to calculate. If we consider scattering based on the object dimension they can be classified into two commonly used scattering mechanisms, namely Rayleigh scattering, where the object dimension is small compared to wavelength $(\sim d \leq 0.1 \lambda)$, and Mie scattering, where the object dimension is large compared to wavelength $(\sim d>10 \lambda)$. Since the system is configured for normal incidence with the sample, the general analysis for oblique incidence is not covered here but is explained in [24]. 


\section{SchottKy Diode-BASEd Device MEASUREMENTS AND ANALYSIS}

The general design methodology for the six-anode Schottky balanced frequency tripler was based on the topology presented in [25]. The fundamental mixer design consisted of a two-anode balanced structure based on the work in [26]. The discrete Schottky diodes used in these designs are fabrication technology developed by Teratech Components Ltd. EM simulations were conducted in Ansoft's HFSS (High Frequency Structure Simulator), while the non-linear circuit simulation was implemented in Keysight's ADS (Advanced Design System. The test benches for measuring the $W$-band frequency tripler and $W$-band fundamental mixer are presented in Figs. 3 and 4, respectively.

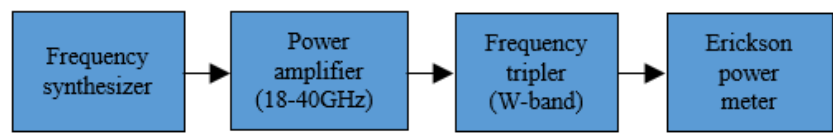

Fig. 3. Block diagram for $W$-band frequency tripler measurement.

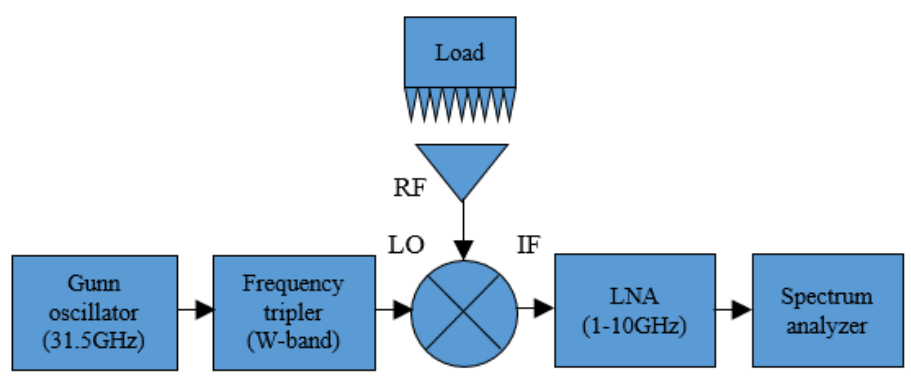

Fig. 4. Block diagram for $W$-band fundamental mixer measurement.

\section{A. W-band Frequency Tripler}

To calibrate the input power to the $W$-band frequency tripler, an $18-40 \mathrm{GHz}$ power amplifier and an Agilent power meter were used. An isolator was placed after the amplifier to prevent standing waves from affecting the measurement. The tripler bias was tuned using an external supply. The output was measured using an Erickson PM4 power meter. Fig. 5 shows the uncorrected room temperature performance of the tripler at optimum bias. A measured conversion efficiency of $8 \%$ was obtained in the $78-102 \mathrm{GHz}$ frequency band for 100 $\mathrm{mW}$ input. The decrease in power at the edges is likely due to impedance matching issues or alignment problems during assembly. Nevertheless, the performance was enough to supply local oscillator (LO) power to the fundamental mixer.

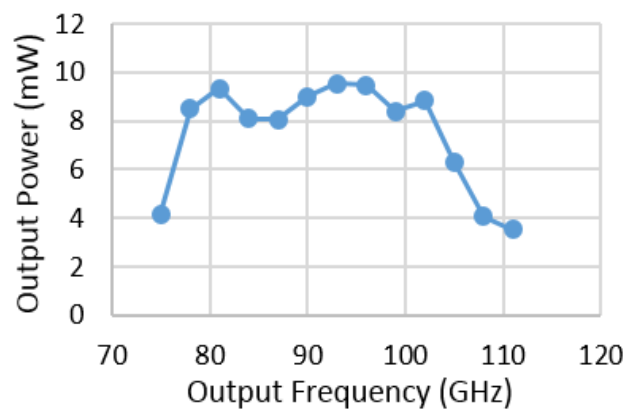

Fig. 5. Measured output power across complete $W$-band for $100 \mathrm{~mW}$ input.

\section{B. W-band Fundamental Mixer}

Standard Y-factor analysis was implemented to measure the $W$-band fundamental mixer performance. An absorber at room temperature, $P_{h o t}$ and another immersed in liquid nitrogen, $P_{\text {cold }}$ were the two targets for finding the Y-factor ratio. An Agilent $346 \mathrm{C}$ noise source was used to calibrate the IF chain. The mixer IF performance was measured using a Rohde \& Schwarz, FSQ26 spectrum analyzer. A Matlab code was implemented for generating the mixer noise temperature, $T_{\text {mix }}$ and conversion gain, $G_{m i x}$ based on the following expression

$$
T_{\text {rec }}=T_{m i x}+\frac{T_{I F}}{G_{m i x}}
$$

where $T_{r e c}$ is the receiver noise temperature and $T_{I F}$ is the IF noise temperature. The measured mixer noise temperature and conversion loss are shown in Fig. 6. The room temperature, double sideband (DSB) noise temperature was generally between 400 and $600 \mathrm{~K}$ with a conversion loss of up to $5 \mathrm{~dB}$. In order to reduce ripples in the measurement, the first LNA at the IF had a low VSWR. The detailed measurement results for the $W$-band fundamental mixer are presented in [27].
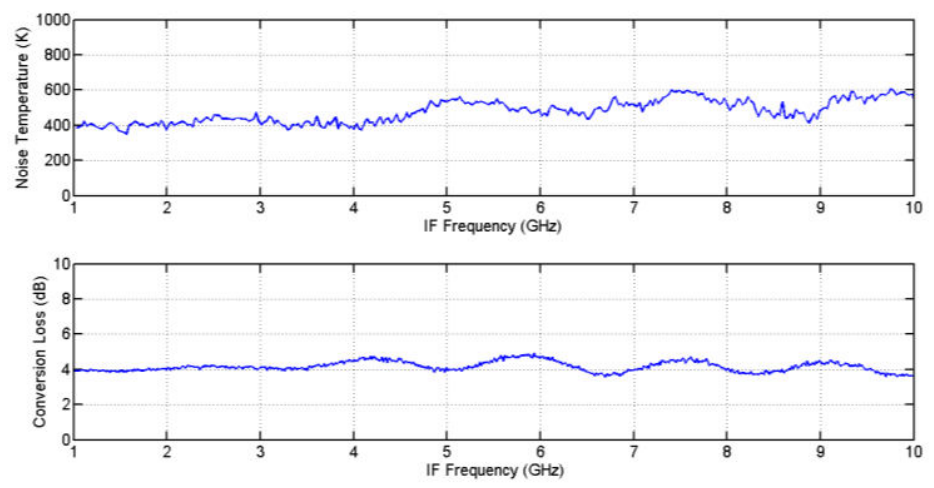

Fig. 6. Ambient noise temperature and conversion loss of $W$-band fundamental mixer centered at $94 \mathrm{GHz}$.

\section{Radiometer Sensitivity}

The radiometer sensitivity was characterized by measuring the noise equivalent temperature difference (NETD) of the system. This value provided an estimate of the minimum signal that could be measured in the presence of noise fluctuations. The sensitivity of a radiometer is defined as the ratio of its RMS noise voltage, $V_{r m s}$ to the temperature sensitivity of the system, $d V / d T$, where $d V$ and $d T$ are the changes in measured voltage and temperature, respectively.

$$
N E T D=\frac{V_{r m s}}{d V / d T}
$$

The radiometer set-up for NETD measurement is shown in Fig. 7. A SR560 low noise preamplifier from Stanford Research Systems and an Agilent DSOX 2024A digital storage oscilloscope (DSO) were also required for the measurement. The zero bias Schottky detector used for signal rectification had a low-level sensitivity of $500 \mathrm{~V} / \mathrm{W}$. 


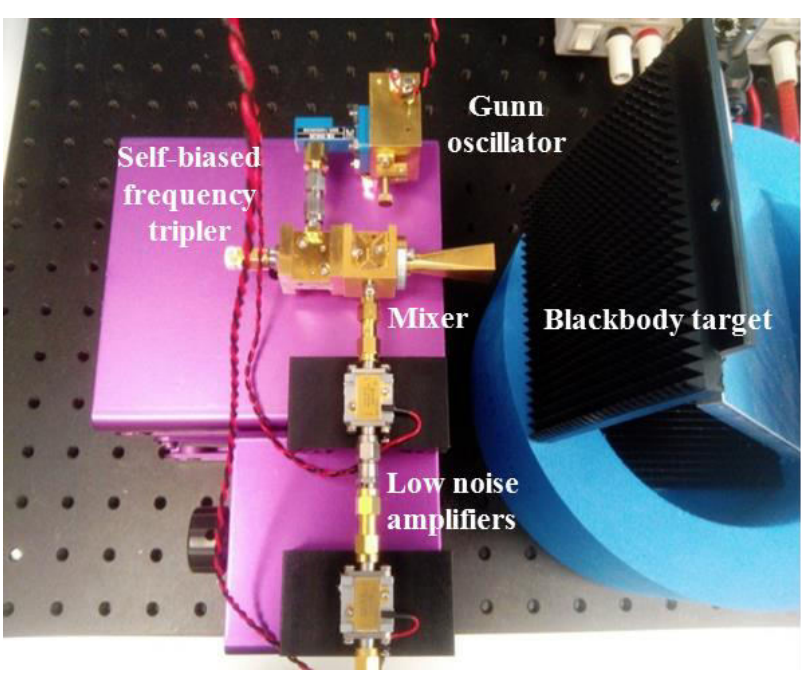

Fig. 7. Set-up for radiometer characterization includes a Gunn source, selfbaiased $W$-band frequency tripler, $W$-band fundamental mixer, and LNAs.

The complete NETD set-up and measurement procedure is given in [28]. The relation between NETD and integration time varies as the inverse square root when the gain variation is small. This can be seen from

$$
N E T D=\left(T_{S}+T_{R}\right)\left[\frac{1}{B \tau}+\left(\frac{\Delta G}{G}\right)^{2}\right]^{1 / 2}
$$

where $\tau$ is the integration time (related to filter bandwidth), $B$ is the RF bandwidth, $T_{R}$ is the receiver noise temperature, $T_{S}$ is the scene temperature and $G$ is the IF amplifier gain. If the gain varies by $\Delta G$, then the sensitivity of the radiometer system can be improved by increasing $B$ and/or decreasing $T_{R}$. Fig. 8 shows the plot for the measured and calculated NETD for the radiometer. The calculated NEDT curve was generated from (19) using $T_{S}=300 \mathrm{~K}, T_{R}=2000 \mathrm{~K}$ and $B=10 \mathrm{GHz}$. By fitting the calculated with the measured results, gain instability of $0.025 \%$ was obtained. Gain fluctuation is usually higher for longer integration time as they have a $1 / f$ power spectrum [29].

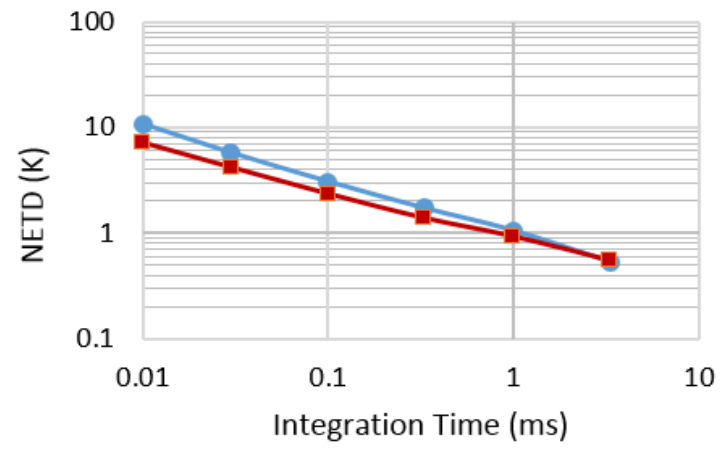

Fig. 8. Measured (round marker) and calculated (square marker) NETD results after accounting for gain instabilities in the measurement.

\section{ACTIVE RAdiometer SySTEM ARCHITECTURE}

The active radiometer system developed for NDT of GFRP can be divided into three main parts; the photomixer-based transmitter for illuminating the sample, a quasi-optical test bench that comprised of polytetrafluoroethylene (PTFE) lenses for focusing the free space signal and the receiver which was a high sensitivity $W$-band Schottky diode-based radiometer.

\section{A. Photomixer}

Broadband mm-wave power was generated by a photomixer driven by optical noise centered around a wavelength of 1.5 $\mu \mathrm{m}$ [30]. The near-infrared power was provided by amplified spontaneous emission (ASE) from an erbium-doped optical fiber amplifier (EDFA). The photomixer consisted of a high speed InGaAs photodiode coupled to a standard rectangular WR10 waveguide. Although the photomixer is normally used to produce a single $W$-Band frequency when driven by two lasers [31], in this case the frequency components within the incoherent light from the EDFA beat with each other to generate a wide mm-wave spectrum. This spectrum was characterized as shown in Fig. 9 using a Fourier transform spectrometer. The sharp spectral features appeared due to variations in impedance match with frequency between the photodiode and waveguide sections. Output power indicated at frequencies below the $59 \mathrm{GHz}$ waveguide cut-off is an artefact arising from the Fourier transformation process.

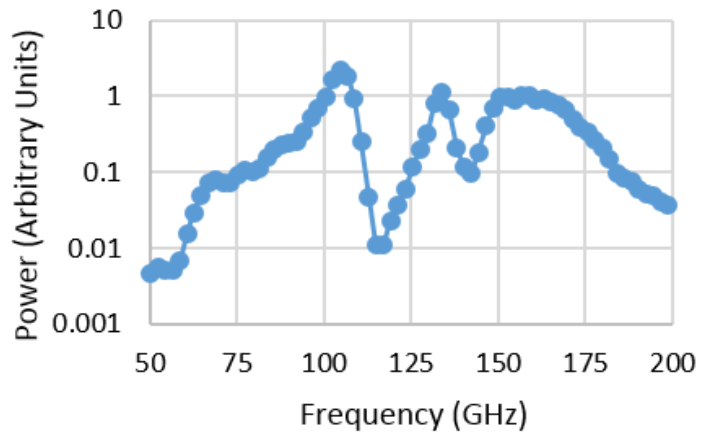

Fig. 9. Output power spectrum from a photomixer diven by optical noise.

The photomixer's output was amplified and passed through a calibrated rotary vane attenuator before being coupled into free space by a pyramidal feedhorn. The illumination system settings were such that a maximum, frequency integrated, illumination power of $1 \mathrm{~mW}$ was available from the measurements described below. The photomixer's broadband output was amplified by a narrow-band power amplifier which had $20 \mathrm{~dB}$ linear gain from 80 to $100 \mathrm{GHz}$. Therefore the $3 \mathrm{~dB}$ bandwidth of the power amplifier was considered as the operational bandwidth of the system.

\section{B. Quasi-Optical Test Bench}

The quasi-optical test bench employed two equal sets of plano-convex PTFE lenses on either side of the sample under test. The two lenses were mounted back-to-back, one for obtaining an electromagnetic plane wave and the other for focusing this plane wave. One set of lenses was used between the transmitter and sample and other between the sample and 
radiometer. The complete test bench is shown in Fig. 10. The plano-convex lenses, separated by the sum of their focal lengths, were arranged to obtain a narrow Gaussian beam waist at the sample. The position of the lenses was verified by a Gaussian beam software, GRASP (Generalized Reflector Antenna Analysis Software Package) developed by TICRA.

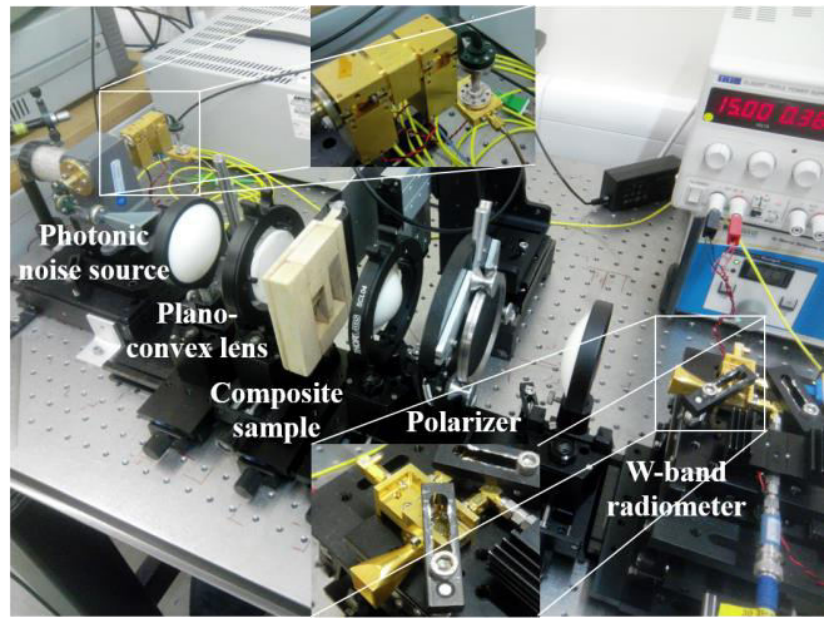

Fig. 10. Measurement test bench includes photomixer noise source (inset), plano-convex lenses, composite sample, polarizer, and $W$-band radiometer (inset).

\section{Radiometer}

The $W$-band radiometer was driven with a fixed tuned Gunn oscillator at $31.5 \mathrm{GHz}$. A Gunn source was chosen for its stability and low noise compared to other sources, such as backward wave oscillators (BWO) [32]. The signal from the oscillator was frequency tripled and supplied as an LO source power of approximately $7 \mathrm{~mW}$ at $94 \mathrm{GHz}$ to the $W$-band fundamental mixer. The IF signal bandwidth was $1-18 \mathrm{GHz}$, but was measured upto $10 \mathrm{GHz}$ due to the unavailability of LNAs with low noise figure (NF) and VSWR to cover the entire frequency band. Fig. 11 shows the LO generation scheme for the radiometer. Signal amplification of the mixer IF was performed using two cascaded 1-10 GHz LNAs. The P1 dB compression points for the LNAs were $12 \mathrm{dBm}$ and 13 $\mathrm{dBm}$, respectively. As per the expression in (17), the receiver noise temperature is heavily dependent on the noise performance of the first LNA at the IF. Hence a LNA with 30 $\mathrm{dB}$ gain, $1.4 \mathrm{~dB} \mathrm{NF}$, and 1.2 VSWR was placed first in the IF chain. The second LNA had a $32 \mathrm{~dB}$ gain, $1.55 \mathrm{~dB} \mathrm{NF}$, and 1.3 VSWR. A digital voltmeter was used to record the varying output voltage due to sample inhomogeneities.

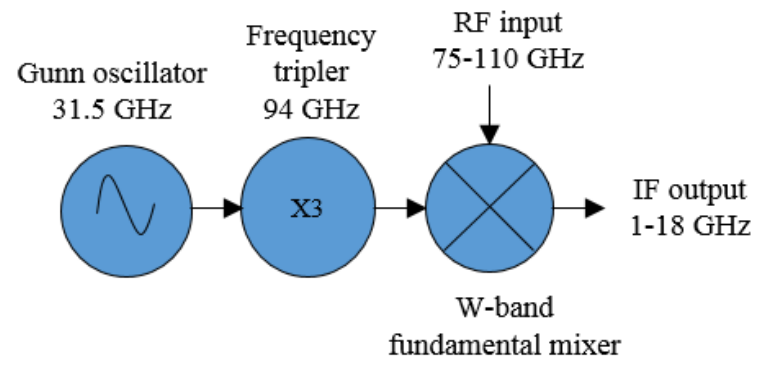

Fig. 11. LO generation scheme for the radiometer centered at $94 \mathrm{GHz}$.

\section{SYSTEM CALIBRATION}

The system calibration involved placement of the planoconvex PTFE lenses on the quasi-optical test bench. The lens immediately after the noise source converted the diverging Gaussian beam into a collimated beam between the two lenses, which was then focused onto the sample. The lenses on the receiver side focused the transmitted diverging signal on the radiometer. To ensure the required focusing was available, 60-mm focal length lenses were selected closer to the sample. The outer lenses had a focal length of $150 \mathrm{~mm}$. A chopper and lock-in amplifier were employed to account for instabilities in the radiometer, however, it was seen that this was not necessary due to high signal to noise and good system stability. This may however prove advantageous for samples with lower transparency or increased signal scattering.

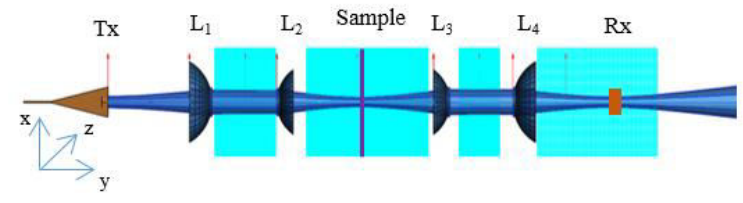

Fig. 12. Quasi-optical Gaussian beam model from GRASP. Tx is the noise source, $\mathrm{Rx}$ is the radiometer and $L_{1}, L_{2}, L_{3}$, and $L_{4}$ are the plano-convex lenses.

(a)

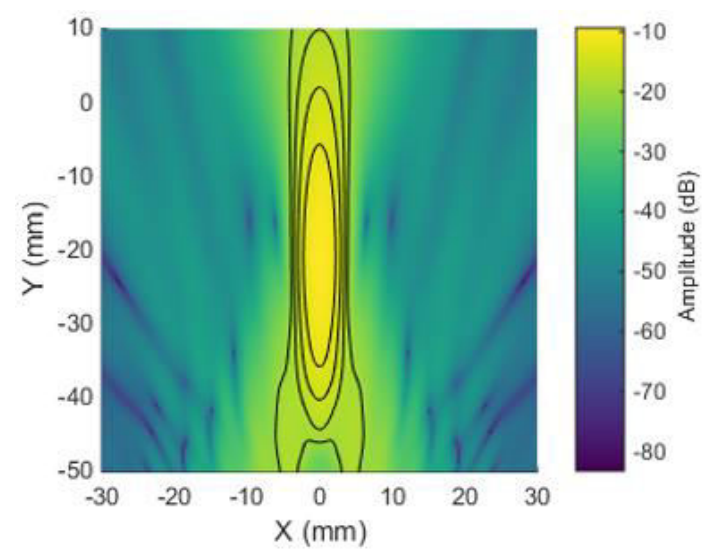

(b)

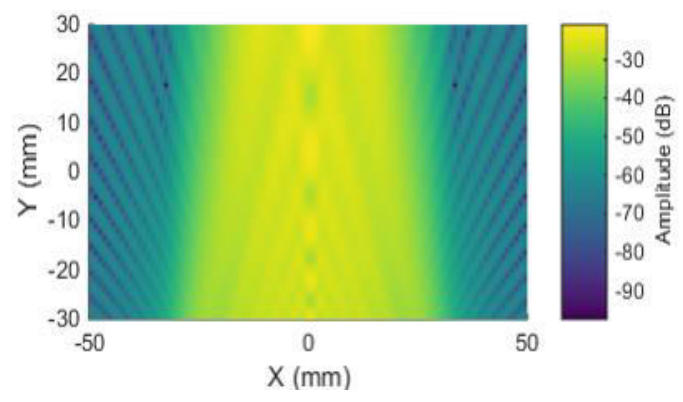

Fig. 13. The $3 \mathrm{~dB}$ Gaussian beam intensity graphs showing (a) converged beam at $\mathrm{Rx}$ (b) parallel beam waist between $L_{3}-L_{4}$.

The lens positions were aligned to maximize the received signal. Fig. 12 illustrates the optics arrangement in GRASP. Beam intensity plots obtained from the simulator are shown in Fig. 13(a), which indicates the beam at the radiometer, while 
Fig. 13(b) shows the parallel beam between the two lenses on the radiometer side, i.e., $L_{3}-L_{4}$. The results show a shift in the beam waist along the direction of propagation of the signal. This shift was due to approximation of the feedhorn and lens parameters which were needed for simulating the optics in GRASP. The spot size determined as describe above was approximately $2.5 \mathrm{~mm}$ in diameter.

\section{Active RAdiometer Imaging Results}

To demonstrate the imaging capability of the system, defects in the form of steps and a wedge with gradually varying depth were machined on a MDF (medium density fiber) sample. Fig. 14 shows the MDF sample used for inspection. The sample had an overall dimension of $70 \times 85 \times$ $25 \mathrm{~mm}^{3}$. Each step was $20 \times 15 \times 5 \mathrm{~mm}^{3}$. The wedge-shaped defect had a dimension of $20 \times 60 \mathrm{~mm}^{2}$ with a maximum depth of $20 \mathrm{~mm}$.

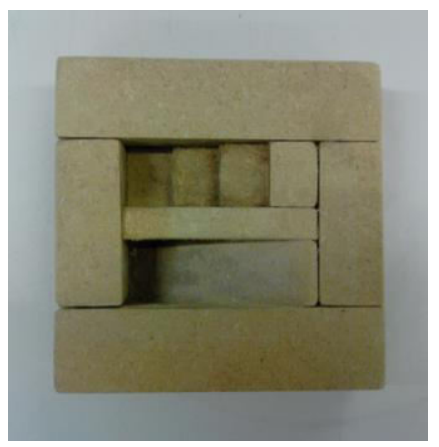

Fig. 14. Photgraph of the machined MDF sample showing step and wedge defects.

Fig. 15 shows the transmission and scattering characteristics from the MDF sample as it was linearly scanned across the center of the step and wedge defects. For this imaging experiment, both the transmitter and receiver were copolarized. The transmission power through the sample was characterized as a voltage signal from the radiometer. The linear scan in Fig. 15(a) clearly shows the transmission signal changing due to varying depths in the step defect. While moving from left to right on the sample, each step increased in height by $5 \mathrm{~mm}$. This corresponds to a decrease in voltage measured at the radiometer for each step. Since there was no change in polarization at the radiometer, signal transmission along non-defected area shows a constant voltage.

A sharp decrease in voltage was seen at the edges of the sample which indicates attenuation of the scattered fields due to change in polarization. The plot in Fig. 15(b) shows a gradual decrease in signal transmission as the sample is scanned from a larger depth (less signal attenuation) to a smaller depth (more signal attenuation). As with the step defects, the two edges on either ends of the wedge showed a decrease in voltage due to scattering of the signal. The difference in voltage levels at these edges is due to the randomness of the source transmission as these levels changed at every scan. From the transmission data obtained in Fig. 15, if a linear map of the output voltage and the sample length is generated it would be possible to estimate depth information of the defects. (a)

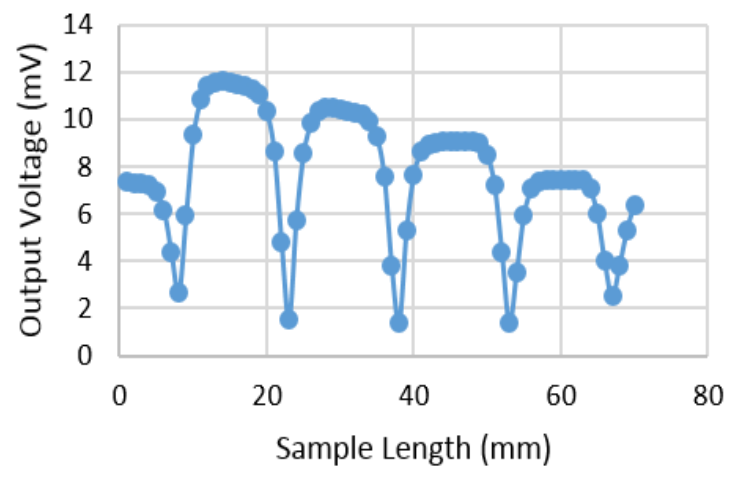

(b)

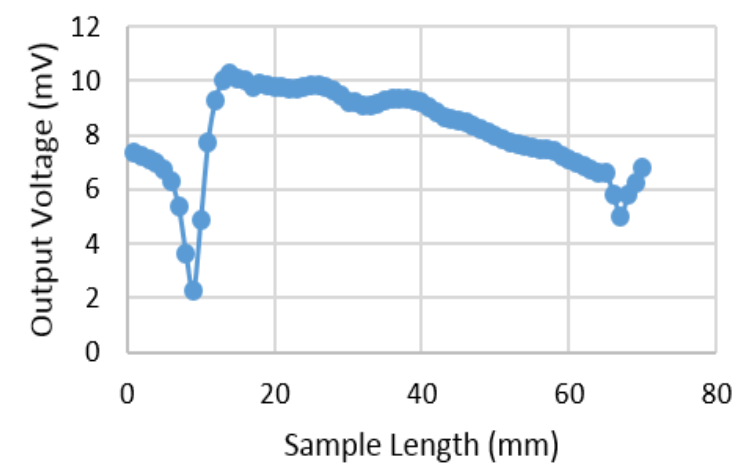

Fig. 15. Linear scans for MDF sample with defects of varying depths. (a) Step defect. (b) Wedge-shaped defect.

The intensity plots for the MDF test sample are shown in Fig. 16. Each step defect illustrated a maximum in transmitted signal along the surface with varying depths, whereas the edges showed a minimum due to scattering. The signal transmission along the non-defected areas was constant throughout the sample. For the wedge-shaped defect, the signal transmission showed a gradual decrease in intensity as it moved towards a smaller depth in the wedge. The transmission characteristics obtained from the sample indicates that it would be possible to estimate the defect density with reasonable accuracy, while the signal scattering would help in identifying cracks and edges in certain samples.

In the interest of correlating the transmission characteristics illustrated in Fig. 16 with theoretical expression, material properties for MDF were substituted in (16). An approximate value of $2.5+j 0.02$ was selected for the relative permittivity.

On solving the equation and taking the integral average across the bandwidth, a decrease of approximately $1.5 \mathrm{~dB}$ in received power was obtained while moving from the $10 \mathrm{~mm}$ thick part to the $20 \mathrm{~mm}$ thick part of the sample. This translated to 1.95 $\mathrm{mV}$ difference in voltage at the detector. To validate imaging results from the MDF sample, signal transmission between the step defects with $10 \mathrm{~mm}$ thickness difference should show a value similar to the detected voltage. On examining the linear scan in Fig. 15(a), the difference between voltage levels for two step defects with $10 \mathrm{~mm}$ thickness difference was approximately $2.2 \mathrm{mV}$. This shows that the imaging results reasonably agree with the transmission theory. 

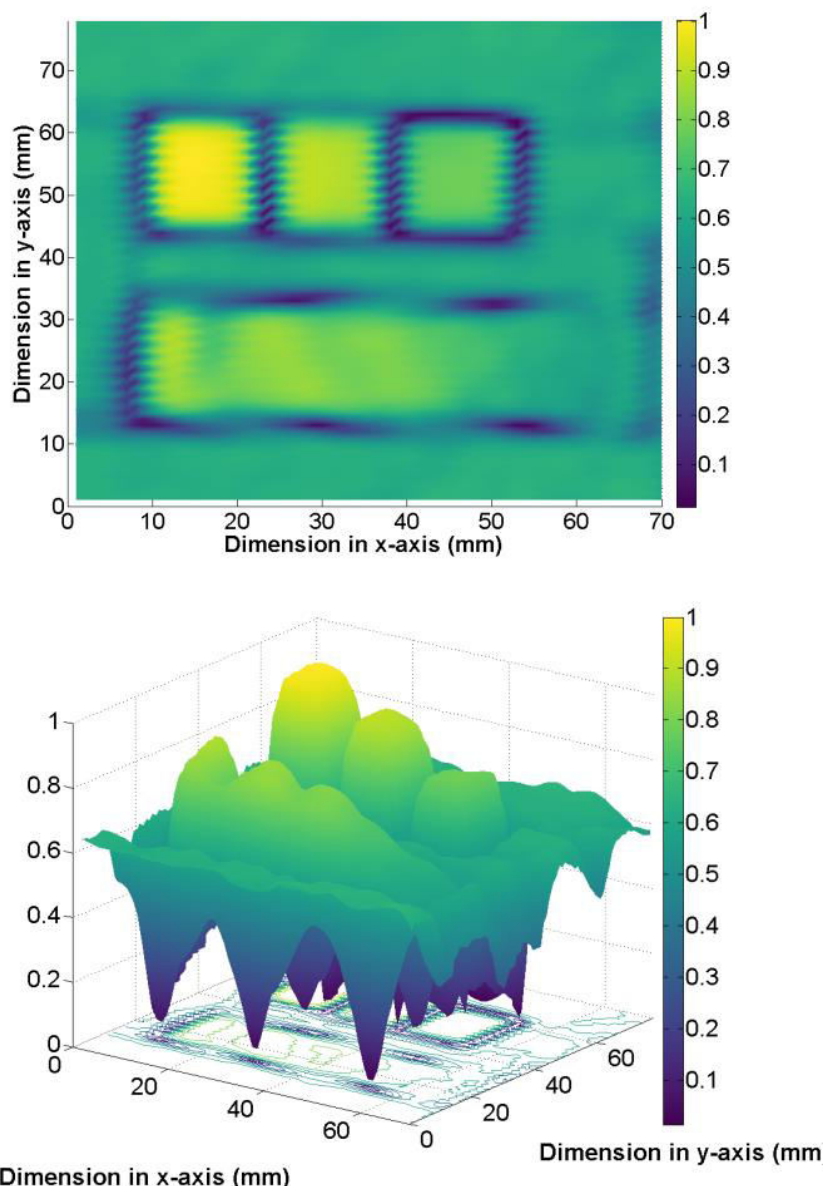

Fig. 16. Intensity profile for MDF sample showing maximum signal trasmission along the defect surface and minimum transmission along the defect edges.

The GFRP sample shown in Fig. 17 was carefully machined to have dimensions of $40 \times 30 \times 5 \mathrm{~mm}^{3}$. Two blind hole defects of 2 and $4 \mathrm{~mm}$ diameters were drilled into the sample, to $3 \mathrm{~mm}$ depth. As the defects on the sample moved into the incident beam of the radiometer a gradual change in output voltage was noted. Since the spontaneous thermal radiation of GFRP sample is low compared to the transmitted photonic noise, the radiometer only sees changes in transmission noise due to the air-dielectric interface. The change in transmission signal intensity across the sample is illustrated in Fig. 18. It is clear from this figure that the right-hand feature is associated with the 4-mm diameter defect. As mentioned earlier, the decrease in signal voltage on approaching a defect was associated with signal scattering from its edges. This conclusion assumes that the large bandwidth of the noise source would overall average out the effects of destructive and constructive interference of the incident and reflected waves from the defect area, leaving the drop in measured transmitted intensity to be due to scattering. However, it is not unlikely that an internal standing wave is present at certain frequencies, which reduces or increases the return loss due to reflections.

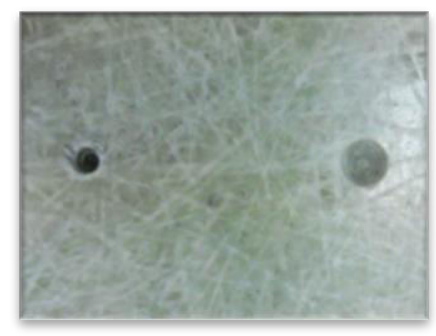

Fig. 17. Photograph of the GFRP sample showing two machined circular defects of $2 \mathrm{~mm}$ and $4 \mathrm{~mm}$ diameters.
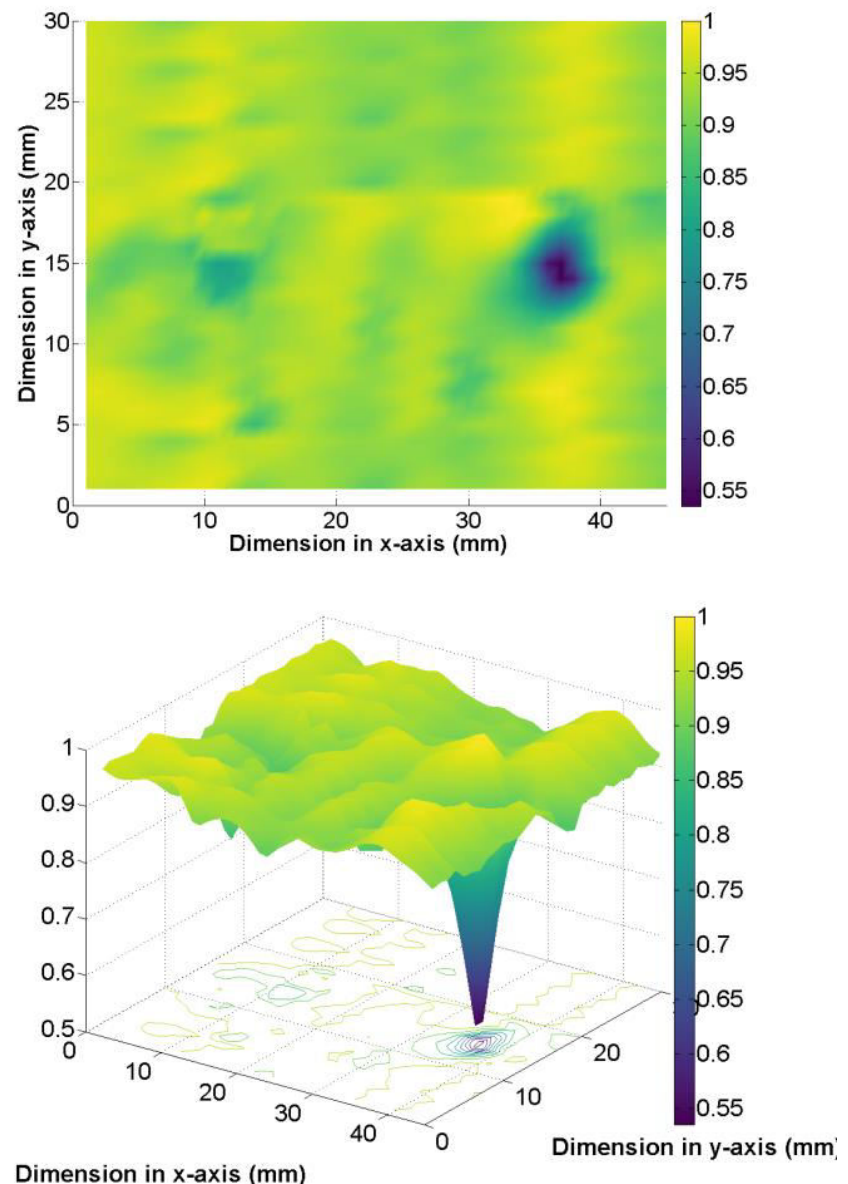

Fig. 18. Intensity profile for the GFRP sample showing maximum and minimum signal transmission.

\section{CONCLUSION}

This work demonstrates the successful application of an active $W$-band radiometer system for imaging composites, particularly a GFRP sample with machined defects as small as a few $\mathrm{mm}$ in diameter. The system described here consisted of a photonic noise source and a high sensitivity Schottky-based radiometer for detecting defects as a change in measured output voltage. The radiometer had a noise equivalent temperature difference of $0.53 \mathrm{~K}$ at $3.33 \mathrm{~ms}$ integration time. A spot size of approximately $2.5 \mathrm{~mm}$ in diameter was obtained using Gaussian beam modelling in GRASP. The linear scans along with the intensity plots for MDF sample demonstrated the imaging capability of the system. The results obtained 
from imaging of the GFRP sample confirmed that active imaging with a radiometer is a viable option for NDT/E. In principle, the active radiometer-based NDT/E system can be compact and fast scanning times achieved due to its simple voltage output for image generation. The attraction of such a system is that it is easy to implement and that it has a small form factor, as demanded by NDT/E inspections in the field. With future integration bringing down manufacturing costs, this makes for a clear path to implementation where other systems do not progress from the laboratory. In practice, a robotic in situ alignment can be envisaged with existing precision robotic positioners.

\section{REFERENCES}

[1] M. Etcheverry and S. E. Barbosa, "Glass Fiber Reinforced Polypropylene Mechanical Properties Enhancement by Adhesion Improvement," Materials, vol. 5, no. 12, pp. 1084-1113, Jun. 2012.

[2] Y. Deng and X. Liu, "Electromagnetic Imaging Methods for Nondestructive Evaluation Applications," Sensors, vol. 11, no. 12, pp. 11774-11808, Dec. 2011.

[3] L. Diener, "Microwave near-field imaging with open-ended waveguideComparison with other techniques of nondestructive testing," Research in Nondestructive Evaluation, Springer-Verlang, June 1995, vol. 7, no. 2, pp. 137-152.

[4] M. Firdaus, R. Sloan, C. I. Duff, M. Wielgat, and J. F. Knowles, "Nondestructive Evaluation of Thermal Barrier Coated Turbine Blades using Microwave Techniques," Materials Evaluation, vol. 74, no. 4, pp. 543-551, April 2016.

[5] L. Cheng, G. Y. Tian, and B. Szymanik, "Feasibility studies on microwave heating for nondestructive evaluation of glass fibre reinforced plastic composites," IEEE International Instrumentation and Measurement Technology Conference (I2MTC), Binjiang, China, 2011, pp. 1-6.

[6] N. N. Qaddoumi, W. M. Saleh, and M. Abou-Khousa, "Innovative NearField Microwave Nondestructive Testing of Corroded Metallic Structures Utilizing Open-Ended Rectangular Waveguide Probes," IEEE Transactions on Instrumentation and Measurement, vol. 56, no. 5, pp. 1961-1966, Oct. 2007.

[7] A. Foudazi, K. M. Donnell, and M. T. Ghasr, "Application of Active Microwave Thermography to delamination detection," IEEE International Instrumentation and Measurement Technology Conference (I2MTC) Proceedings, Montevideo, Uruguay, 2014, pp. 1567-1571.

[8] D. K. Ghodgaonkar, N. A. Ali, and L. Giubbolini, "Microwave nondestructive testing of composite materials using free-space microwave measurement techniques," 15th World Conference on Non-Destructive Testing, Roma, Italy, Oct. 2000, pp. 15-21.

[9] B. M. Abdullah, J. Cullen, A. Mason, A. I. Al-Shamma'a, C. J. Hawkins, and M. Saunders, "An investigation into the potential of microwave NDT for structural materials," Sixth International Conference on Sensing Technology (ICST), Kolkata, India, 2012, pp. 464-469.

[10] M. Nezadal, J. Schür, and L. P. Schmidt, "Non-destructive testing of glass fibre reinforced plastics with a full polarimetric imaging system," in 39th International Conference on Infrared, Millimeter, and Terahertz waves (IRMMW-THz), Tucson, AZ, USA, 2014, pp. 1-2.

[11] O. Büyüköztürk and Tzu-Yang Yu, "Far-field radar NDT technique for detecting GFRP debonding from concrete," Construction and Building Materials, vol. 23, no. 4, pp. 1678-1689, April 2009.

[12] G. Dobmann, I. Altpeter, C. Sklarczyk, and R. Pinchuk, "NonDestructive Testing with Micro- and MM-Waves - Where We are Where We Go," Welding in the World, vol. 56, no. 1, pp. 111-120, Jan. 2012.

[13] F. Rutz, S. Wietzke, M. Koch, H. Richter, S. Hickmann, V. Trappe, and U. Ewert, "Non-Destructive Testing of Glass-Fibre Reinforced Polymers using Terahertz Spectroscopy," 9th European Conference on NonDestructive Testing, Berlin, Germany, 2006, pp. 1-8.

[14] C. Ibarra-Castanedo, A. Bendada, N. P. Avdelidis, and X. P. V. Maldague, "Nondestructive assessment of glass fibre composites by midwave and near infrared vision," Materials Transactions, vol. 53, no. 4, pp. 601-603, Feb. 2012.

[15] T. Clancy, F. Ulaby, and C. Dobson, "Detection of Space Shuttle tile defects using millimeter-wave radiometry," IEEE Antennas and
Propagation Society International Symposium, Salt Lake City, UT, USA, vol.3, 2000, pp. 1787-1790.

[16] S. Bakhtiari, N. Gopalsami, and A. C. Raptis. "Determining bonding quality in polymer composites with a millimeter wave sensor," Review of Progress in Quantitative Nondestructive Evaluation, Springer US, 1997, pp. 665-671.

[17] T. Shibuya, Y. Goto, A. Dobroiu, C. Otani, and K. Kawase, "Nondestructive detection of carbon in soot collection filters by using a 94 $\mathrm{GHz}$ source," Joint 32nd International Conference on Infrared and Millimeter Waves and the 15th International Conference on Terahertz Electronics, Cardiff, UK, 2007, pp. 273-274.

[18] K. H. Seah, M. F. Karim, L. C. Ong, and T. M. Chiam, "Rapid corrosion detection using $94 \mathrm{GHz}$ millimeter wave technology," IEEE International Instrumentation and Measurement Technology Conference (I2MTC), Graz, Austria, 2012, pp. 473-476.

[19] S. W. Harmer, S. Shylo, M. Shah, N. J. Bowring, and A. Y. Owda, "On the feasibility of assessing burn wound healing without removal of dressings using radiometric millimetre-wave sensing," Progress in Electromagnetics Research M, vol. 45, pp. 173-183, Jan. 2016.

[20] S. Bakhtiari, T. Elmer, N. M. Cox, N. Gopalsami, A. Raptis, S. Liao, I. Mikhelson, and A. Sahakian, "Compact Millimeter-Wave Sensor for Remote Monitoring of Vital Signs," IEEE Transactions on Instrumentation and Measurement, vol. 61, no. 3, pp. 830-841, March 2012.

[21] F. T. Ulaby, R. K. Moore, A. K. Fung, Microwave Remote Sensing Active and Passive - Microwave Remote Sensing Fundamentals and Radiometry, vol. 1, Addison-Wesley, Reading, 1981, pp. 344-418.

[22] L. C. Shen and J. A. Kong, "Reflection and Transmission of Waves," in Applied Electromagnetics, $3^{\text {rd }}$ ed., Boston, MA, USA: Cengage Learning, 1995, pp. 84-103.

[23] N. Ida, "Reflection, Transmission, and Scattering of Waves," Microwave Ndt, Dordrecht: Kluwer, 1992, pp. 103-151.

[24] S. Ramo, J. R. Whinnery, T. V. Duzer, "Plane-Wave Propagation and Reflection," Fields and Waves in Communication Electronics, $3^{\text {rd }}$ ed.J. Wiley, New York, 1994, pp. 300-313.

[25] A. Maestrini, C. Tripon-Canseliet, J. Ward, H. Javadi, J. Gill, G. Chattopadhyay, E. Schlecht, and I. Mehdi, "Multiple-Anode Broadband Frequency Tripler at Submillimeter Wavelengths," Proceeding of the Sixteenth International Symposium on Space Terahertz Technology, paper P5-3, Goteborg, Sweden, 2-4 May 2005.

[26] B. Thomas, A. Maestrini, J. Gill, C. Lee, R. Lin, I. Mehdi, P. de Maagt, "A Broadband 835-900-GHz Fundamental Balanced Mixer Based on Monolithic GaAs Membrane Schottky Diodes," IEEE Transactions on Microwave Theory and Techniques, vol. 58, no. 7, pp. 1917-1924, June 2010.

[27] C. Viegas, B. Alderman, J. Powell, H. Liu, H. Wang and R. Sloan, "Millimeter wave radiometers for applications in imaging and nondestructive testing," 8th UK, Europe, China Millimeter Waves and THz Technology Workshop (UCMMT), Cardiff, UK, 2015, pp. 1-4.

[28] C. Viegas, B. Alderman, J. Powell, H. Liu, H. Wang and R. Sloan, "Characterization of $94 \mathrm{GHz}$ and $183 \mathrm{GHz}$ planar schottky diode based radiometer modules," IEEE MTT-S International Microwave and RF Conference (IMaRC), Hyderabad, India, 2015, pp. 296-299.

[29] N. R. Observatory. (Oct. 2016). Radiometers Internet. [Online]. Available: http://www.cv.nrao.edu/course/astr534/Radiometers.html

[30] P. G. Huggard, L. Azcona, B. N. Ellison, P. Shen, N. J. Gomes, and P. A. Davies, "Application of $1.55 \mu \mathrm{m}$ photomixers as local oscillators \& noise sources at millimetre wavelengths," Int. Conf. Infrared Millimetre Waves, Karlsruhe, Germany, 2004, pp. 771-772.

[31] P. G. Huggard, B. N. Ellison, P. Shen, N. J. Gomes, P. A. Davies, W. P. Shillue, A. Vaccari, and J. M. Payne, "Efficient generation of guided millimeter-wave power by photomixing," IEEE Photonics Technology Letters, vol. 14, no. 2, pp. 197-199, Feb. 2002.

[32] K. Dahlberg, T. Kiuru, J. Mallat, T. Narhi, and A. V. Raisanen, "MixerBased Characterization of Millimeter-Wave and Terahertz Single-Anode and Antiparallel Schottky Diodes," IEEE Transactions on Terahertz Science and Technology, vol. 4, no. 5, pp. 552-559, June 2014. 


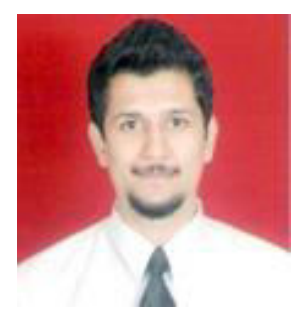

Colin Viegas received the B.E. degree in Electronics and Telecommunication Engineering from the University of Mumbai, India, in 2009. Thereafter, he worked at Society for Applied Microwave Electronics Engineering and Research (SAMEER), Mumbai from 2009 to 2011. He received the M.Sc. degree in Communication Engineering from The University of Manchester, UK, in 2012 and is currently a Ph.D. student at the university. His research is in collaboration with Teratech Components Ltd. where he works on Schottky based millimeter and sub-millimeter wave components for local oscillators and mixers.

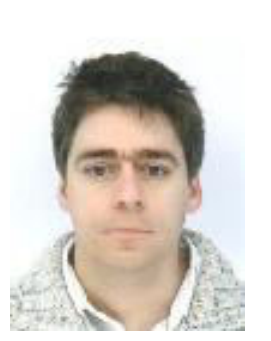

Byron Alderman received his MPhys degree in Physics from the University of Warwick, U.K., in 1998, and his Ph.D. from the University of Leeds, U.K., in 2001. Following graduation he joined the Millimetre Wave Technology Group at the Rutherford Appleton Laboratory, UK. He founded Teratech Components Limited in 2010 and currently the CEO of Teratech and a Principal Scientist at the Rutherford Appleton Laboratory. His research interests are in the field of room temperature heterodyne receiver technology for applications in remote sensing and astronomy at millimetre and sub-millimetre wavelengths.

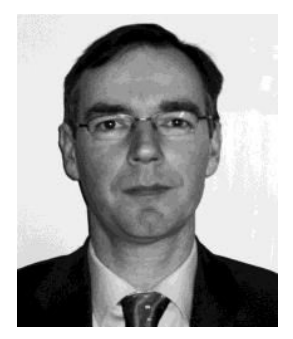

Peter Huggard received BA(Mod) in Experimental Physics (First Class Honours) and $\mathrm{PhD}$ degrees from the University of Dublin, Trinity College in 1986 and 1991 respectively. He then spent three years at the Universitaet Regensburg, Germany, working on high power molecular vapour infrared lasers and non-linear interactions of infrared radiation with condensed matter. From 1995 to 2000 he was at the Physics Department of the University of Bath, developing a THz time domain spectroscopy system for the study of bulk and two dimensional semiconductors. Since 2000, Dr Huggard has been a member of the Millimetre Wave Technology Group in the UK's STFC Rutherford Appleton Laboratory. As a Principal Scientist within STFC, he has developed and provided the fibre optic coupled photomixer sources which generate the phase locking signals for the ALMA radio telescope. Dr Huggard's current research interests include developing a range of sources and detectors for $\mathrm{THz}$ radiation.

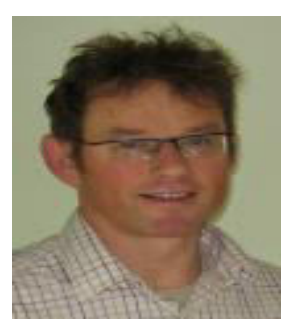

Jeff Powell received $\mathrm{BSc}$ and $\mathrm{PhD}$ degrees from the University of Birmingham in the UK in 1992 and 1995 respectively. Following graduation he continued work at Birmingham investigating properties of ferroelectric and superconducting materials at microwave frequencies. From 2001 to 2010 he worked as a principal engineer at QinetiQ in the UK where he performed many MMIC circuit, hybrid and module designs for many applications from 2 to $110 \mathrm{GHz}$ using a wide range of commercial and research-based circuit and packaging technologies. In 2010 he formed Skyarna Ltd, a design consultancy which specialises in the design of leading edge circuits; including wideband high efficiency amplifiers and active circuits to $300 \mathrm{GHz}$. He is currently working with Teratech Components on technology development. He has contributed to over 50 journal and conference publications and also 2 patent applications.

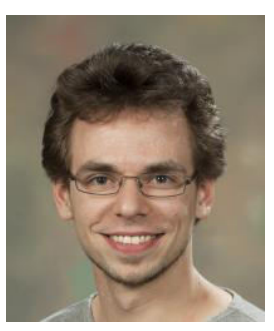

Kai Parow-Souchon received his MSc in Electronics Engineering from RWTH Aachen University, Germany, in 2014. He then joined the Millimetre Wave Group at the Rutherford Appleton Laboratory, UK. He has worked on design and test of large quasi-optical networks as well as millimetre wave component design.

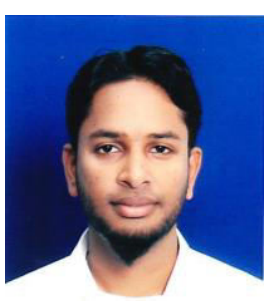

Muhammad Firdaus A. J. K (GSM'16) was born in Penang, Malaysia, in 1986. He received the B.Sc. and M.Sc. degrees in Communication engineering from the International Islamic University Malaysia and University of Manchester, in 2010 and 2012, respectively. From 2010 to 2011, he was a R\&D Engineer at Motorola Solutions in Penang. From 2012 to 2014, he was an Electrical Engineer at Usains Infotech Sdn Bhd in Penang. He is currently a Research Student with the University of Manchester, Manchester, U.K. His research interests include electromagnetics and microwave non-destructive testing (NDT).

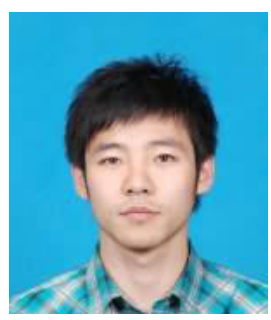

Hairui Liu PhD, obtained his Doctorate at Beijing University of Post and Telecommunication (BUPT). From 2010 to 2011, he worked at RAL as a visiting scientist. He is now doing post doctor in BUPT. His main research is on millimeter wave components and devices. Hairui Liu has (co-)authored more than 10 journal and conference publications and one patent.

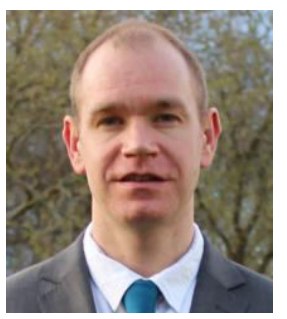

Christopher I. Duff (M'02) received the M.Eng. degree in Electronic Engineering from the University of Manchester Institute of Science and Technology (UMIST) and the Ph.D. degree from the University of Manchester in 2000 and 2005 respectively. He has worked for Roke Manor Research, Romsey, U.K., Creo Medical, U.K. and EADS Astrium, U.K. He is currently a Lecturer in the School of Electrical and Electronic Engineering at the University of Manchester. His current research interests include nonlinear semiconductor device modeling, microwave and millimeter-wave non-destructive test/evaluation (NDT/E) and development of RF and microwave electronics for electrosurgical devices. 


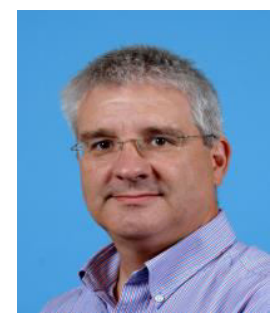

Robin Sloan (M'92-SM'05) received the B.Sc. degree in Electronic Engineering from Sussex University in 1985 and the M.Sc. and Ph.D. degrees from the University of Manchester Institute of Science and Technology (UMIST), Manchester, U.K., in 1988 and 1991, respectively. He has held a number of microwave electronic appointments including working for British Aerospace Air Weapons Division, Hatfield, U.K.; Milmega Limited, Ryde, U.K. and Ferranti International, Poynton, Cheshire, U.K. In July 1994, he was appointed as a Lecturer at UMIST; that subsequently became the School of Electrical and Electronic Engineering in the University of Manchester. He was promoted to Reader in 2007. He was instrumental in setting up the Agilent Millimeter Wave Laboratory in 2004, and since 2007 he has been a Visiting Research Fellow to Keysight Technologies Inc., Santa Rosa, CA, USA. In May 2014 he was awarded a Royal Society Industrial Fellowship and is a co-founder of Microwave Inspection Technologies Limited. $\mathrm{He}$ is an Associate Editor of IET Microwaves, Antennas and Propagation. His current research interests include millimeterwave electronics, device characterization, on-wafer calibration techniques, electromagnetics and microwave NDT. 